\title{
Avaliação da Técnica de Amostragem Estratificada Adaptativa para Identificação de Fluxos "Elefantes" em Redes Convergentes
}

\author{
Vinícius T. Guimarães, Gléderson L. dos Santos, Mateus O. Caruccio, Roberto T. Costa Filho, \\ Ricardo Balbinot, Jorge G. Silveira e José Augusto de Oliveira Neto
}

\begin{abstract}
Resumo - A medição passiva, em especial a medição de tráfego baseada em fluxos, caracteriza-se por prover informações de grande relevância no processo de monitoramento, através da análise do tráfego passante. Entretanto, com o aumento na capacidade das tecnologias de transmissão em rede, o processamento de todos os pacotes que transpassam o ponto de observação acaba se tornando proibitivo, implicando em um problema principal: escalabilidade. Sob essa perspectiva, o presente trabalho apresenta o desenvolvimento e avaliação da técnica de amostragem aleatória estratificada adaptativa aplicada à identificação de grandes fluxos, também denominados fluxos 'Elefante".

Palavras-chave- Medição Passiva. Amostragem aleatória estratificada. Estatísticas de fluxos. Fluxos "Elefante".

Abstract- The passive measurement, especially the traffic based on flows measurement, gives relevant information about monitoring process, through passing traffic analysis. However, with the increase in the transmission capacity of networks, monitoring every packet on a measurement point may no longer be feasible, implicating in a main problem: scalability. In this way, the present work shows the development and evaluation of stratified random adaptive packet sampling technique to identify large or "Elephant" flows.
\end{abstract}

Keywords- Passive Measurements. Stratified Random Sampling. Flows statistics. Elephant flows.

\section{INTRODUÇÃO}

Os esforços no contexto de redes de comunicação fundamentadas no modelo IP têm sido, na última década, direcionados fortemente para as demandas relacionadas aos sistemas de gerenciamento. Neste sentido, Xu et. al. [1] enfatiza que a medição e monitoramento do tráfego são indispensáveis para o gerenciamento de Quality of Service (QoS), planejamento de recursos e projeto de infra-estrutura da rede. Adicionalmente, Estan e Varghese [2] destacam que as informações de medição são essenciais para o monitoramento em curto prazo, engenharia de tráfego em

\footnotetext{
Vinícius T. Guimarães, Gléderson L. dos Santos, Mateus O. Caruccio e Jorge G. Silveira, Grupo de Pesquisa Avançada em Redes de Comunicação e Tecnologia da Informação (GPARC\&TI), Pontifícia Universidade Católica do Rio Grande do Sul, Porto Alegre, RS, Brasil. E-mails \{vicoguim, glederson, mateus.caruccio\}@gmail.com, jorge.guedes@ pucrs.br.

Roberto T. Costa Filho, Ricardo Balbinot e José Augusto de Oliveira Neto, Vice-Presidência de Tecnologia de Rede / Diretoria de Planejamento e Tecnologia (VPTR/DPT), Rio de Janeiro, RJ, Brasil. E-mails \{jose.neto, roberto.iraja, rbalbinot\} @ vivo.com.br. Este trabalho foi realizado com recursos do CNPq, CAPES e VIVO Brasil.
}

longo prazo e contabilização baseada em uso.

Sob esta perspectiva, duas técnicas de medição destacam-se na obtenção de indicadores de desempenho da rede: medição passiva e medição ativa. A medição ativa propõe-se a obter indicadores de desempenho a partir da transmissão controlada e tráfego de teste através da rede que se deseja analisar [3]. Por outro lado, a técnica de medição passiva não requer a injeção de tráfego adicional para mensuração da rede operacional. Dentre as principais informações coletadas a partir desta técnica, pode-se destacar a classificação e contabilização do tráfego baseada em fluxos.

Entretanto, com o aumento na capacidade das tecnologias de transmissão em rede, a classificação e quantificação do tráfego em fluxos, através do processamento de todos os pacotes que transpassam o ponto de observação, acabam tornando-se proibitivas. Segundo [4], as informações de fluxo são tipicamente armazenadas em software e a capacidade de processamento não acompanha a capacidade de transmissão dos enlaces. Além disso, a análise de todos os pacotes contemplando a atualização, armazenamento e exportação das informações de fluxo, requer um alto poder de processamento, capacidade de memória cache, requisições de I/O e consumo de largura de banda.

Partindo deste cenário, observa-se, claramente, que a medição de tráfego baseada em fluxos apresenta como problema principal a falta de escalabilidade. Estudos realizados no final da década de noventa [5][6], os quais utilizaram traces de diferentes backbones, já indicavam que o número de fluxos entre pares de hosts no período de uma hora atingia a marca de 1,7 milhões. Na atualidade, é factível inferir que estes valores cresceram drasticamente.

Endereçando estas restrições, a aplicação de técnicas para amostragem de pacotes apresenta-se como uma aproximação capaz de solucionar os problemas acima apresentados. No Internet Engineering Task Force (IETF), os grupos de trabalho PSAMP [7] e IPFIX [8] recomendam fortemente em suas especificações o uso de amostragem de pacotes. A Request For Comment (RFC) 3176 [9] propõe o sFlow como tecnologia embarcada em roteadores e switches para monitoramento do tráfego utilizando amostragem. Além disso, diferentes fabricantes como Juniper e Cisco já implementam em seus dispositivos métodos estáticos de amostragem, como por exemplo: "amostrar $1 \mathrm{em} \mathrm{N}$ ".

Trabalhos atuais [4][10][11][12] focam na aplicação de 
técnicas de amostragem para a identificação de grandes fluxos ou fluxos "Elefante". O direcionamento para este tipo de fluxo é justificado em [13][14], os quais demonstram a prevalência do fenômeno do "rato" e do "elefante" para fluxos definidos em diferentes níveis de granularidade: um pequeno percentual dos fluxos, tipicamente, contabiliza um grande percentual do tráfego total. Em [10] é mostrado que, em um dos traces analisados, cerca de $0.02 \%$ do total de fluxos contribuíram em mais de $59 \%$ do volume total do tráfego. Desta forma, para muitas aplicações de monitoramento e medição, prover estatísticas acuradas apenas dos fluxos elefantes é suficiente.

Observando esta tendência, o presente trabalho apresenta o desenvolvimento e avaliação da técnica de amostragem aleatória estratificada adaptativa na identificação de fluxos "elefantes", proposta por [4][11]. Basicamente, são apresentadas as principais peculiaridades relacionadas à implementação de um sistema on-line de medição utilizando a técnica de amostragem estratificada adaptativa e a avaliação dos resultados obtidos nos testes realizados, averiguando a fidedignidade das estimas para a contabilização de pacotes e do volume de bytes, em relação à medição tradicional.

$\mathrm{O}$ artigo foi organizado como segue: o segundo capítulo apresenta um resumo da técnica de amostragem estratificada adaptativa proposta por [4][11]. O terceiro capítulo apresenta as principais particularidades relacionadas ao desenvolvimento do sistema de medição utilizando a técnica de amostragem. O quarto capítulo apresenta os testes realizados, sendo os resultados e discussões apresentados no quinto capítulo. O sexto capítulo apresenta as conclusões do trabalho.

\section{AMOSTRAGEM ESTRATIFICADA ADAPTATIVA}

$\mathrm{Na}$ técnica de amostragem estratificada adaptativa[4][11] um fluxo "elefante" é determinado em termos da contabilização de pacotes. Desta forma, os fluxos são classificados como "elefante" baseando-se na proporção da contabilização de pacotes no intervalo de tempo que abrange a duração total do fluxo. Partindo desta premissa, é aplicada a aproximação de estratificação no tempo, ou seja, dividi-se o período de medição em intervalos pré-determinados e não sobrepostos, chamados de estratos ou blocos de tempo.

Assim, para cada bloco de tempo, os pacotes são selecionados com a mesma probabilidade. Sob o ponto de vista de cada fluxo, a sua duração é dividida, ou estratificada, em um tempo fixo, permitindo a estimativa de seu volume, sem conhecer previamente sua dinâmica de chegada e de duração. Partindo de tais premissas, a definição formal de um fluxo elefante é então formalizada, como segue: "considerando-se um intervalo de tempo quantificado que contenha a duração completa de um fluxo $f$. Supondo-se, ainda, que este intervalo seja constituído de $L$ blocos consecutivos, onde um total $m_{i}$ de pacotes são observados no bloco $i(i=1 \ldots L)$ e $m^{f}$ os pacotes pertencentes ao fluxo $f$ dentre o total de pacotes $m$, então, diz-se que um fluxo é "elefante" (1), se a proporção da contabilização de pacotes para o fluxo $p^{f}$ é maior que um limiar $p^{\theta}$ pré-estabelecido".

$$
\frac{m^{t}}{m}=\frac{\sum_{i=1}^{L} m_{i}^{t}}{\sum_{i=1}^{L} m_{i}}=p^{t} \geq p^{\theta}
$$

Para cada fluxo "elefante" identificado, torna-se necessário fazer à estimativa da contabilização do total de pacotes $\hat{m}^{f}$ e a estimativa da contabilização do total de bytes $\hat{v}^{f}$ para o fluxo. Para tal, é preciso limitar o erro relativo da estimativa. $\mathrm{Ou}$ seja, dado um nível de tolerância de erro pré-estabelecido, $\{\eta, \mathcal{\varepsilon}\}$, (onde $(1-\eta)$ e $\varepsilon$ referem-se à confiabilidade e precisão, respectivamente, e $0 \leq \eta \leq 1$ ), a estimação de erro para a contabilização de pacotes e bytes deve ser limitada, de acordo com a seguinte expressão (2):

$$
\operatorname{Pr}\left\{\left|\frac{\hat{m}^{f}-m^{f}}{m^{f}}\right|>\varepsilon\right\} \leq \eta \mathrm{e} \operatorname{Pr}\left\{\left|\frac{\hat{v}^{f}-v^{f}}{v^{f}}\right|>\varepsilon\right\} \leq \eta
$$

Onde $p^{f} \geq p^{\theta}$ para o fluxo $f$.

\section{A. Determinação do número requerido de amostras}

Para determinação do número mínimo de amostras requeridas em cada estrato, a técnica em questão parte das seguintes premissas: o tamanho da amostra, dada por $n$, é suficientemente grande $(n>30)$ e o tamanho da população, dada por $m$ (população), é muito grande quando comparada com o tamanho da amostra $n: m>>n$, de tal forma que a fração de amostragem seja pequena.

Sob estas condições, o Teorema do Limite Central (TLC) é aplicável, ou seja, a distribuição amostral da média tem distribuição normal com média $\mu$ e desvio padrão $\sigma / \sqrt{n}$, independentemente da distribuição da população, sendo $\mu$ e $\sigma$ a média da população e o desvio padrão da população, respectivamente. É conveniente destacar que as amostras são independentes e identicamente distribuídas (i.i.d), conforme a condição imposta pelo TLC e obtidas de forma simples, utilizando-se basicamente uma amostragem aleatória a partir de uma população comum. Assim, a contagem de pacotes para um fluxo é estimada como segue: considera-se um intervalo unitário de tempo que contenha a duração completa de um fluxo $f$, no qual $m$ pacotes são observados; a partir $\operatorname{dos} m$ pacotes observados, $n$ pacotes são aleatoriamente amostrados $(n<m)$, resultando em $n^{f}$ pacotes pertencentes ao fluxo $f$.

Assim, a contabilização de pacotes para o fluxo $f, m^{f}$, é estimada por $\hat{m}^{f}$ utilizando a proporção de amostragem $\hat{p}^{f}$, conforme a equação apresentada a seguir (3):

$$
\hat{m}^{f}=m \cdot \frac{n^{f}}{n}=m \cdot \hat{p}^{f}
$$

A proporção pode ser considerada como um caso especial da média aritmética, onde uma variável $I$ possui apenas os valores 0 e 1 . Então o número de pacotes pertencentes ao fluxo $f$ é $m^{f}=\sum_{i=1}^{m} I_{i}$. Desta forma, no interior de um bloco de tempo (estrato), os pacotes são amostrados com probabilidade fixa (igualmente prováveis). Assim sendo, de acordo com o 
Teorema do Limite Central (TLC) para amostras aleatórias quando $n \rightarrow \infty$, a média da amostra $\hat{p}^{f}$ se aproxima da média da população $p^{f}$ e a variância de $\sigma_{\hat{p}^{f}}^{2}=\frac{p^{f} \cdot\left(1-p^{f}\right)}{n}$, independentemente da distribuição da população. Desta forma, a proporção amostral pode ser escrita em termos da média e variância (4):

$$
\hat{p}^{f} \approx p^{f}+\frac{\sqrt{p^{f} \cdot\left(1-p^{f}\right)}}{\sqrt{n}} \cdot Y_{P}=\mu_{P}+\sigma_{P} \cdot Y_{P}
$$

Onde $Y_{p} \sim N(0,1)$ é a função de densidade de probabilidade padronizada para a estimativa da contagem de pacotes. Assim (2) pode ser reescrita da seguinte forma (5):

$$
\begin{aligned}
& P\left\{\left|\frac{m \hat{p}^{f}-m p^{f}}{m p^{f}}\right|>\varepsilon\right\}=P\left\{\left|\frac{\hat{p}^{f}-p^{f}}{\sigma_{\hat{p}^{f}}}\right|>\frac{p^{f} \sqrt{n} \varepsilon}{\sqrt{p^{f}\left(1-p^{f}\right)}}\right\} \\
& \approx 2\left(1-\Phi\left(\frac{\sqrt{p^{f}} \sqrt{n} \varepsilon}{\sqrt{\left(1-p^{f}\right)}}\right)\right) \leq \eta
\end{aligned}
$$

Onde $\Phi(\cdot)$ é a função de distribuição cumulativa (c.d.f. cumulative distribution function) da distribuição normal padrão. Resolvendo a desigualdade em (5) explicitando-se $n$, é então possível derivar o número mínimo de amostras requeridas $n^{*}, p$ para estimar a contabilização de pacotes dentro de um nível pré-estabelecido de tolerância para o erro $\mathcal{\varepsilon}(6)$ :

$$
n \geq n^{*, p}=\left[z_{p} \cdot\left(\frac{1-p^{f}}{p^{f}}\right)\right]=\left[z_{p} \cdot C_{\theta}\right]
$$

Onde $z_{p}=\left(\frac{\Phi^{-1}(1-\eta / 2)}{\varepsilon}\right)^{2}$ e $C_{\theta}=\frac{1-p^{\theta}}{p^{\theta}}$ são constantes. Desta forma, com um número aleatório mínimo de $n^{*}, p$ amostras, uma amostragem aleatória simples pode fornecer uma confiabilidade pré-especificada $\{\eta, \varepsilon\}$ para qualquer proporção de fluxo que seja maior que um limite estabelecido, $p^{\theta}$, considerado como fluxo "elefante". Para a contabilização do volume de bytes, o número de amostras requeridas para um determinado fluxo pode ser obtida de forma similar a estimação da quantidade de pacotes, conforme (7):

$$
n \geq n^{*, b, f}=\left[z_{p} \cdot\left(\frac{1-p^{f}+S^{f}}{p^{f}}\right)\right]
$$

Onde $s^{f}=\left(\frac{\sigma^{2}}{\mu^{f}}\right)^{2}$ é o SCV (quadrado do coeficiente de variação) do tamanho dos pacotes pertencentes ao fluxo $f$. Conseqüentemente, o número de amostras requeridas, dentre um limite de erro pré-estabelecido, para a estimação do volume de bytes pode ser escrita da seguinte forma (8):

$$
n \geq n^{*, b}=\left[z_{p} . B_{\theta}\right]
$$

Onde $B_{\theta=}\left(\frac{1-p^{\theta}+S^{\theta}}{p^{\theta}}\right)$.

\section{B. Predição do total de pacotes}

Tendo o número requerido de amostras computadas, a probabilidade para um bloco produzir $n^{*}\left(n^{*}, p\right.$ ou $\left.n^{*, b}\right)$ amostras é $P_{s p}=n^{*} / m_{h}$, onde $m_{h}$ é o número total de pacotes pertencentes ao bloco $h$. O termo $n^{*}$ pode ser $n^{*}, p$ para estimar apenas a quantidade de pacotes, ou $n^{*}, b$, para o caso de estimar, adicionalmente, o volume de bytes. Entretanto, não se consegue escolher por uma taxa de amostragem precisa quando o tamanho da população é desconhecido. Para solucionar este problema, a técnica sugere a aplicação de um modelo Auto-Regressivo $\mathrm{AR}(1)$ de série temporal para predizer o tráfego total em termos do número de pacotes $(m)$ para o próximo bloco, utilizando 5 valores. Assim, para finalizar o processo de estimativas, a atualização das estatísticas dos fluxos ativos utilizando a taxa de amostragem no final do bloco $h$ é dada da seguinte forma (9):

$$
\hat{m}_{h}^{f}=\hat{m}_{h-1}^{f}+\frac{m_{h}}{n_{h}} \hat{n}_{h}^{f}, \quad \hat{v}_{h}^{f}=\hat{v}_{h-1}^{f}+\frac{m_{h}}{n_{h}} \hat{n}_{h}^{f} \hat{\mu}_{h}^{f}
$$

\section{DESENVOLVIMENTO}

Basicamente, para a caracterização do tráfego em fluxos, baseou-se na abordagem usual, onde um fluxo é determinado por uma tupla composta por cinco atributos: endereço IP de origem, endereço IP de destino, número da porta de origem, número da porta de destino e número de protocolo. Além disso, para cada fluxo são coletadas as seguintes informações: contabilização do número de pacotes e volume de bytes.

Para agregar os mecanismos inerentes a técnica de amostragem em questão, tornou-se necessário realizar algumas adaptações no sistema tradicional de medição de fluxos, adicionando seis parâmetros complementares: "block_time" representa o intervalo de duração em segundos que cada bloco (estrato) deverá conter; "threshold" $\left(p^{\ominus}\right)$ limiar que indica se um determinado fluxo é ou não elefante, devendo estar no intervalo $0 \leq p^{\theta} \leq 1$; "past_predict" número de blocos passados a serem utilizados na predição do próximo bloco, pelo modelo $\operatorname{AR}(1)$; "reliability" $(\eta)$ corresponde a confiabilidade das estimativas, segundo um nível de tolerância de erro préestabelecido devendo estar no intervalo $0 \leq \eta \leq 1$; "precision" $(\varepsilon)$ : corresponde a precisão das estimativas, segundo um nível de tolerância de erro pré-estabelecido devendo estar no intervalo $0 \leq \varepsilon \leq 1$; " $s c v "\left(S^{\ominus}\right)$ coeficiente que indica a variabilidade do tamanho dos pacotes que compõem um fluxo elefante, assumindo valores no intervalo $0,00007 \leq S^{\theta} \leq 1[4]$.

\section{A. Predição do total de pacotes para o próximo bloco}

Conforme apresentado anteriormente, a probabilidade de amostragem é computada no começo de um novo bloco (estrato), através da predição do total de pacotes. Porém, quando o sistema é inicializado não existem valores passados para a predição ser realizada. Para tal, determinou-se um período de convergência. O período de convergência (em segundos) é calculado através do produto do valor informado 
para o parâmetro past_predict +1 pelo valor informado para o parâmetro block_time, consirerando as especificações do modelo AR(1). No encerramento do período de convergência, é realizado o primeiro processo de predição para o próximo bloco e, a partir do número de pacotes estimados, é realizada a seleção aleatória dos pacotes. Na predição propriamente dita, é empregada regressão linear e para estabelecimento dos parâmetros $a$ e $b$ previstos no modelo são aplicadas às fórmulas apresentadas abaixo [15]:

$$
\begin{aligned}
& m_{h}=a+b \cdot m_{h-1} \\
& b=\frac{S P X Y}{S Q X}, \text { onde }:\left\{\begin{array}{l}
S P X Y=\sum_{j=1}^{n}\left(X_{j}-\bar{X}\right)\left(Y_{j}-\bar{Y}\right) \\
S Q X=\sum_{j=1}^{n}\left(X_{j}-\bar{X}\right)^{2} \\
a=Y_{\text {med }}-b \cdot X_{\text {med }}
\end{array}\right.
\end{aligned}
$$

Onde $m_{h}$ é o valor futuro (predito); $n$ é o número de estados passados; $X$ e $Y$ são vetores de $n$ elementos.

A seleção das amostras para o bloco seguinte foi implementada utilizando o número de pacotes preditos como base para a definição do espaço amostral. Basicamente, os números aleatórios são sorteados no intervalo $\left[1 ; m_{h}\right]$, onde $m_{h}$ é o número de pacotes estimados pelo sistema de predição.

\section{B. Divisão e processamento dos blocos}

A partir do valor informado para a configuração do parâmetro block_time é instanciado um processo auxiliar, que se torna responsável por gerar um sinal a cada vez que um bloco de tempo (estrato) é completado. Segundo esta abordagem, tornou-se necessário criar adaptações no processo de classificação do tráfego em fluxos, assim como para contabilização do total de pacotes e do volume de bytes.

De acordo com a modelagem da técnica de amostragem, as estimativas das informações dos fluxos são realizadas no final de cada bloco, ou seja, o real preenchimento da tabela de fluxos deve ser realizado, analogamente, no final de cada estrato. Para tratar esta especificidade, utilizou-se um mecanismo de tabela de fluxos temporária.

Basicamente, no decorrer de cada bloco é criada uma tabela temporária de fluxos a partir dos pacotes amostrados. O preenchimento desta tabela segue os mesmo critérios do sistema de medição tradicional. Assim, no final do bloco têmse as informações desejadas: o total de pacotes observados no bloco (total de pacotes independente de fluxos) e a classificação do tráfego em fluxos com base nos pacotes amostrados. Com posse dessas informações a tabela temporária de fluxos é atualizada, recebendo o valor das estimativas calculadas para a contabilização de pacotes e do volume de bytes para cada fluxo. Após o encerramento do processo de atualização da tabela temporária de fluxos, cada entrada que compõe esta tabela passa a ser atualizada na tabela global de fluxos atualizando também um campo adicional que contém o somatório do total de pacotes observados nos blocos pelos quais o fluxo perdurou.

$\mathrm{Na}$ rotina de migração das informações contidas na tabela temporária de fluxos para a tabela global de fluxos utilizam-se os mesmos critérios. Ou seja, caso um novo fluxo seja observado no interior do bloco, esta entrada também irá se consolidar como uma nova entrada na tabela global de fluxos. Por outro lado, para o caso de fluxos anteriormente observados, a tabela global já terá uma entrada contendo a mesma chave identificadora, bastando atualizar as informações.

\section{TESTES REALIZADOS}

Para a avaliação da técnica de amostragem em questão foram realizados diferentes procedimentos de teste objetivando averiguar as divergências oriundas dos resultados obtidos com o sistema de medição utilizando a abordagem tradicional em relação ao sistema utilizando amostragem, para a contabilização de pacotes e volume de bytes. Os testes foram realizados utilizando um modelo de cenário de rede real controlado, implementado em laboratório. No presente trabalho, são abordados dois conjuntos de procedimentos de testes, onde ambos utilizaram a transmissão de fluxos de interesse, empregando-se valores empíricos para os parâmetros requeridos pela técnica de amostragem. Para a transmissão dos fluxos de interesse, foi utilizada a transferência de arquivos sob o protocolo HyperText Transfer Protocol (HTTP).

$\mathrm{O}$ primeiro conjunto objetivou observar o comportamento das estimativas utilizando apenas fluxos de interesse, transmitidos de forma paralela não-sincronizada e sem a existência de tráfego competitivo. Os fluxos de interesse tinham as seguintes características: 1 Gbytes, 800 Mbytes, 500 Mbytes, 100 Mbytes, 50 Mbytes, 10 Mbytes, 1 Mbytes, 500 Kbytes. A Tabela 1 apresenta os parâmetros utilizados para os procedimentos realizados no primeiro conjunto de teste.

$$
\text { TABELA I }
$$

PARÂMETROS UTILIZADOS NO PRIMEIRO CONJUNTO DE TESTES.

\begin{tabular}{cccccccc}
\hline \hline & $\eta$ & $\mathcal{E}$ & $P^{\theta}$ & $S^{\theta}$ & $n^{*, b}$ & past_predict & Estrato \\
\hline P1 & 0.1 & 0.1 & 0.01 & 0.2 & 32196 & 5 & $10 \mathrm{~s}$ \\
P2 & 0.1 & 0.1 & 0.01 & 0.2 & 32196 & 5 & $20 \mathrm{~s}$ \\
P3 & 0.1 & 0.1 & 0.01 & 0.2 & 32196 & 5 & $30 \mathrm{~s}$ \\
P4 & 0.2 & 0.2 & 0.01 & 0.2 & 4886 & 5 & $10 \mathrm{~s}$ \\
P5 & 0.2 & 0.2 & 0.01 & 0.2 & 4886 & 5 & $20 \mathrm{~s}$ \\
P6 & 0.2 & 0.2 & 0.01 & 0.2 & 4886 & 5 & $30 \mathrm{~s}$ \\
P7 & 0.3 & 0.3 & 0.01 & 0.2 & 1420 & 5 & $10 \mathrm{~s}$ \\
P8 & 0.3 & 0.3 & 0.01 & 0.2 & 1420 & 5 & $20 \mathrm{~s}$ \\
P9 & 0.3 & 0.3 & 0.01 & 0.2 & 1420 & 5 & $30 \mathrm{~s}$ \\
\hline
\end{tabular}

O segundo conjunto foi concebido a partir dos diagnósticos provenientes do primeiro conjunto e com o intuito de averiguar os resultados das estimativas na existência de tráfego competitivo. Os fluxos de interesse tinham as seguintes características: 1 Gbytes, 800 Mbytes, 500 Mbytes, 100 Mbytes, 500 Kbytes, 100 Kbytes, 5 Kbytes. O tráfego competitivo foi injetado no cenário de teste utilizando o aplicativo udpflood (desenvolvido pela própria equipe para realização dos testes). A Tabela 2 apresenta a descrição dos parâmetros para o segundo conjunto. 
TABELA II

PARÂMETROS UTILIZADOS NO SEGUNDO CONJUNTO DE TESTES.

\begin{tabular}{cccccccc}
\hline \hline & $\eta$ & $\mathcal{E}$ & $P^{\theta}$ & $S^{\theta}$ & $n^{*, b}$ & past_predict & Estrato \\
\hline P1 & 0.1 & 0.1 & 0.01 & 0.2 & 32196 & 5 & $10 \mathrm{~s}$ \\
P2 & 0.2 & 0.2 & 0.01 & 0.2 & 4886 & 5 & $10 \mathrm{~s}$ \\
P3 & 0.3 & 0.3 & 0.01 & 0.2 & 1420 & 5 & $10 \mathrm{~s}$ \\
\hline
\end{tabular}

\section{Resultados E Discussões}

Constatou-se com relação ao primeiro conjunto de testes a influência do tamanho dos estratos na fidedignidade das estimativas. Este fato se torna evidente no ambiente de testes utilizado, pois com o aumento no período de duração dos estratos, o tamanho da população aumentou proporcionalmente. Este comportamento no crescimento do tamanho da população é bastante particular do tipo de tráfego empregado.

O uso do HTTP, para transferências dos fluxos de interesse, trouxe consigo as particularidades do protocolo Transmission Control Protocol (TCP), especificamente, o controle de fluxo. Esta característica, determinou que o tamanho da população fosse crescendo gradativamente com o aumento no tamanho dos estratos, uma vez que o controle de fluxo TCP ajusta o tamanho da janela de transmissão em razão das condições da rede. Desta forma, embora a transmissão dos fluxos de interesse tenha sido realizada de forma paralela e nãosincronizada, a capacidade de transmissão sempre esteve próxima do limiar máximo.

Outro ponto a ser destacado no primeiro conjunto de procedimentos é relativamente mais visível e está relacionado aos valores atribuídos para os coeficientes $\eta$ e $\varepsilon$, confiabilidade e precisão das estimativas, respectivamente. A utilização de diferentes valores para estes coeficientes teve como objetivo verificar o comportamento da técnica de amostragem com a diminuição do número de amostras.

Neste contexto foi observado o comportamento esperado, ou seja, com a diminuição no número de amostras, juntamente com o aumento no tamanho dos estratos, o percentual de erro foi mais elevado. $\mathrm{O}$ erro mais significativo nas estimativas aconteceu para o fluxo de 500 Kbytes, ultrapassando $100 \%$, no procedimento 9 (P9). Este comportamento é plausível, uma vez que este fluxo não foi classificado como "Elefante". Para os grandes fluxos, o percentual de erro chegou a atingir $18 \%$, no procedimento $8(\mathrm{P} 8)$, para o fluxo de 1 Gbyte.

Os resultados obtidos a partir do primeiro conjunto de teste foram preponderantes para entender alguns aspectos intrínsecos a medição utilizando amostragem. Neste sentido, no segundo conjunto de procedimentos de testes, utilizou-se o intervalo de duração para os estratos que se mostrou mais adequado segundo o modelo de tráfego empregado, variando apenas os coeficientes $\eta$ e $\varepsilon$. Além disso, a presença de tráfego competitivo tinha por objetivo manter o ambiente de teste mais próximo de um ambiente real. A adição dos fluxos de 100 Kbytes e de 5 Kbytes foi efetuada com o intuito de manter um contraponto mais acentuado entre o número de fluxos "elefante" e de pequenos fluxos. Nos três procedimentos de teste a média do total de pacotes em cada bloco foi de
168130,6 pacotes. A taxa de amostragem média em cada um dos procedimentos de testes do segundo conjunto é apresentada na Tabela 3 .

TABELA III

TAXA DE AMOSTRAGEM MÉDIA NO SEGUNDO CONJUNTO DE TESTES.

\begin{tabular}{cccc}
\hline \hline & $n^{*, b}$ & Estrato & Média da taxa de amostragem (\%) \\
\hline P1 & 32196 & $10 \mathrm{~s}$ & $19,2 \%$ \\
P2 & 4886 & $10 \mathrm{~s}$ & $2,9 \%$ \\
P3 & 1420 & $10 \mathrm{~s}$ & $0,8 \%$ \\
\hline
\end{tabular}

Com base nas informações apresentadas, os resultados obtidos com o uso da técnica de amostragem nos procedimentos do segundo conjunto de testes mostraram-se satisfatórios. A fidedignidade das estimativas para os fluxos "elefante", especificamente nos procedimentos de teste 1 e 2 (P1 e P2), é plenamente aceitável, dado o percentual de pacotes amostrados. Especialmente no procedimento $2(\mathrm{P} 2)$, onde a média da taxa de amostragem consolidou-se em torno de $2.9 \%$ do total de pacotes em cada bloco, pode-se observar exatidão nas estimativas tanto do total de pacotes como do volume de bytes, sendo que, no pior caso, o percentual de erro não excedeu $2.3 \%$. Exemplificando, a Fig. 1, a Fig.2 e a Tabela 4 apresentam o comportamento nos três procedimentos para o fluxo de 800 Mbytes.

Comparativo para o fluxo de $800 \mathrm{MB}$

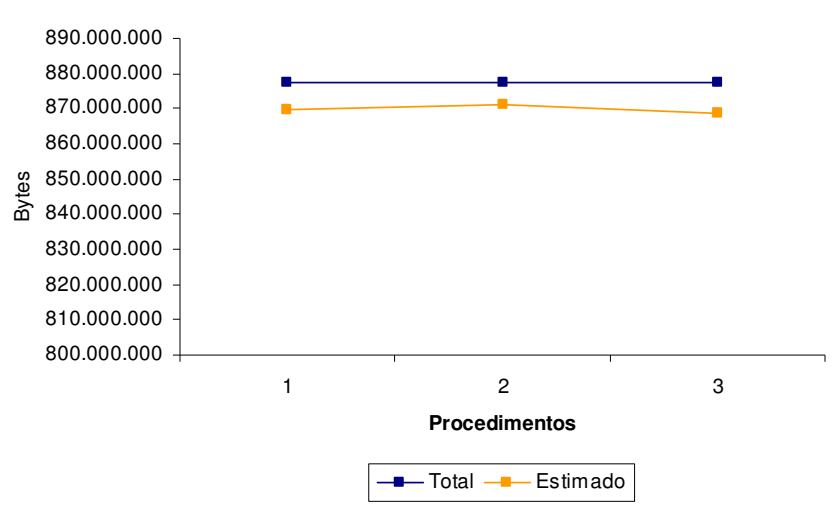

Fig. 1. Comportamento na estimativa de bytes para o fluxo de 800 Mbytes.

Comparativo para o fluxo de $800 \mathrm{MB}$

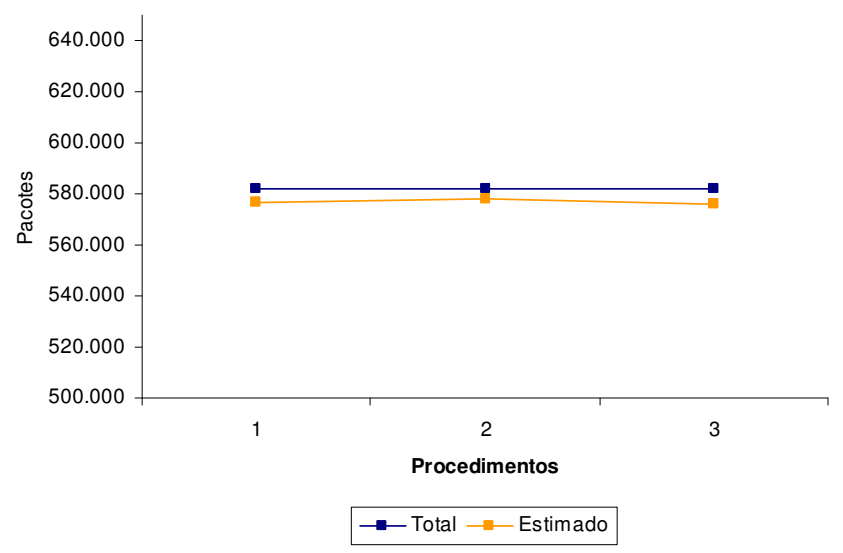

Fig. 2. Comportamento na estimativa de pacotes para o fluxo de 800 Mbytes. 
TABELA IV

RESULTADOS PARA O FLUXO DE $800 \mathrm{MBYTES}$

\begin{tabular}{ccccccc}
\hline \hline & \multicolumn{3}{c}{ Bytes } & & \multicolumn{3}{c}{ Pacotes } \\
\cline { 2 - 7 } P1 & Total & Estimado & IErrol & Total & Estimado & |Errol \\
\cline { 2 - 7 } P2 & 877253534 & 869895095 & $0,8 \%$ & 581792 & 576920 & $0,8 \%$ \\
P3 & 877179000 & 868843341 & $1 \%$ & 581825 & 576319 & $0,9 \%$ \\
\hline
\end{tabular}

Para o procedimento 3 (P3), o percentual de erro começa a apresentar valores mais significativos. Essa perda nas estimativas se apresenta de forma pontual, ou seja, não se distribui pelos fluxos, fato que induz a inferência de que a mínima variação na dinâmica de transmissão dos fluxos pode gerar distorções localizadas. Além disso, pelos parâmetros configurados para este procedimento, no qual a taxa de amostragem foi reduzida significativamente, era esperado que os resultados das estimativas apresentassem divergências mais acentuadas.

De outro lado, as estimativas para os pequenos fluxos apresentaram-se bastante deturpadas nos três procedimentos, fato que demonstra a coerência no foco em grandes fluxos para o delineamento estatístico da técnica de amostragem empregada. A medição dos pequenos fluxos neste contexto, assim como no primeiro conjunto de testes, só foi realizada com o objetivo de ratificar este comportamento, pois em um ambiente real de medição os pequenos fluxos seriam descartados automaticamente, desalocando memória na tabela de fluxos e minimizando o transporte e armazenamento das informações de fluxo.

\section{CONCLUSÕES}

No presente trabalho foi apresentado o desenvolvimento e a avaliação dos resultados obtidos com a implementação da técnica de amostragem adaptativa em um sistema de medição on-line para redes convergentes. Por tratar-se de um tema que ainda germina no cenário acadêmico-científico, as proposições ainda estão no campo de pesquisa. Por este motivo, tornou-se necessária a inserção de mecanismos particulares para obtenção de um protótipo capaz de reproduzir as características especificadas pela técnica de amostragem em questão, assim como para manter os requisitos requeridos para um sistema de medição on-line.

No que tange da técnica de amostragem aleatória estratificada adaptativa, observou-se que a mesma deve ser empregada com base em um conhecimento prévio do comportamento usual da rede. Essa constatação foi extraída com base nos testes realizados, uma vez que variações significativas nos parâmetros de configuração, utilizando um mesmo ambiente de teste, apresentam, analogamente, variações bastante representativas nas estimativas obtidas.

Especificamente, observou-se que o intervalo de duração definido para o estrato pode gerar distorções nos resultados. Desta forma, o dimensionamento deste parâmetro deve ser realizado, primeiramente, com base na capacidade do enlace que está sendo mensurado. Este dimensionamento pode ser realizado de forma empírica, uma vez que o objetivo é minimizar possíveis perdas na fidedignidade das estimativas simplesmente por falta de critério e coerência na parametrização do sistema de medição.

Seguindo neste contexto, observou-se nos testes realizados que, quando parametrizado de forma condizente com o ambiente a ser mensurado, o sistema de medição utilizando a técnica de amostragem apresenta um erro percentual, para fluxos considerados "elefante", que não ultrapassa $3 \%$ nas estimativas de contabilização do total de pacotes e volume de bytes. Este resultado mostra que a técnica de amostragem estratificada adaptativa, apresenta resultados plenamente aceitáveis para aplicação em diferentes contextos. Pelo erro percentual obtido, a utilização é factível até mesmo em cenários mais críticos como, por exemplo, a averiguação de quebra de cláusulas contratuais de um SLA.

\section{REFERÊNCIAS}

[1] L. Xu, G. Wu, J. Li, "Packet-Level Adaptive Sampling on MultiFluctuation Scale Traffic," Proceedings Communications, Circuits and Systems, v. 1, p. 604-608, Agosto 2005.

[2] C. Estan, K. Keys, D. Moore, G. Varghese, "Building a Better NetFlow," Proceedings of the conference on Applications, technologies, architectures, and protocols for computer communications, p. 245-256, 2004.

[3] G. Santos, V. Guimarães, J. Silveira, A. Vieira, J. A. Neto, R. Costa, R. Balbinot, "UAMA: a Unified Architecture for Active Measurements in IP Networks," Proceedings of the IFIP/IEEE International Symposium on Integrated Network Management. Maio 2007, to apeear.

[4] B. Choi, J. Park, Z. Zhang, "Adaptive Packet Sampling for Accurate and Scalable Flow Measurement," IEEE Communications Society, Dezembro 2004.

[5] K. Thompson, G. J. Miller, R. Wilder, "Wide-area Internet traffic patterns and characteristics," IEEE Network, v. 11, p. 10-23, Dezembro 1997.

[6] W. Fang, L. Peterson, "Inter-AS traffic patterns and their implications," IEEE Global Telecommunications Conference, v. 3, p. 1859-1868, Maio 1999.

[7] J. Quittek, "Packet Sampling (psamp)," IETF charter. 2006.

[8] D. Plonka, N. Brownlee, "IP Flow Information Export (ipfix)," IETF charter. 2007.

[9] P. Phaal, S. Panchen, N. Mckee, "InMon Corporation's sFlow: A Method for Monitoring Traffic in Switched and Routed Networks," IETF - RFC 3176, Setembro 2001.

[10] T. Mori, M. Uchida, R. Kawahara, "Identifying Elephant Flows Through Periodically Sampled Packets," Proceedings of the 4th ACM SIGCOMM conference on Internet measurement, p. 115-120. 2004.

[11] B. Choi, Z. Zhang, "Adaptive random sampling for traffic volume measurement," Telecommun Syst, Springer Science. Dezembro 2006.

[12] C. Estan, G. Varghese, "New Directions in Traffic Measurement and Accounting: Focusing on the Elephants, Ignoring the Mice," $A C M$ Transactions on Computer Systems, v. 21, p. 270-313, Agosto 2003.

[13] Y. Zhang, L. Breslau, V. Paxson, S. Shenker, "On the Characteristics and Origins of Internet Flow Rates," Proceedings of ACM SIGCOMM, p. 309-322, Agosto 2002.

[14] A. Feldmann, N. Greenberg, N. Reingold, C. Lund, J. Rexford, F. True, "Deriving traffic demands for operational IP networks: Methodology and Experience," Transactions on Networking, IEEE/ACM, Pág. 265 279, Junho 2001.

[15] A. P. Pires, "Notas de séries temporais," LMAC, IST - Instituto Superior Técnico. Lisboa. 2001. 